\title{
Erratum
}

pISSN: 2234-6163 • elSSN: 2234-6171

https://doi.org/10.5999/aps.2019.01256• Arch Plast Surg 2019;46:491

\section{Clinical outcomes of a low-cost single-channel myoelectric-interface three-dimensional hand prosthesis}

\author{
Inhoe $\mathrm{Ku}^{1}$, Gordon K. Lee ${ }^{2}$, Chan Yong Park ${ }^{3}$, Janghyuk Lee ${ }^{4}$, Euicheol Jeong ${ }^{5}$ \\ ${ }^{1}$ Department of Plastic and Reconstructive Surgery, Seoul National University Hospital, Seoul, Korea; ${ }^{2}$ Division of Plastic and Reconstructive \\ Surgery, Stanford Medical Center, Stanford, CA, USA; ${ }^{3}$ Department of Trauma Surgery, Wonkwang University Hospital, Iksan; ${ }^{4}$ Beauty \\ Plastic Surgery Clinic, Seoul; ${ }^{5}$ Department of Plastic Surgery, SMG-SNU Boramae Medical Center, Seoul, Korea
}

Arch Plast Surg 2019;46:303-10. https://doi.org/10.5999/aps.2018.01375

We have noticed two errors in our published paper above.

\section{Erratum: Correction of Figure}

In Fig. 3, the name of "Figure by courtesy of Sanho Yi" should be "Sangho Yi."

\section{Erratum: Correction of Table}

In Table 2, the P-value of VAS score in the Pre-Post 3rd should be revised from 0.03 to 0.3.

The revised table is shown below.

\section{Table 2. OPUS-UEFS scores, VAS scores, and prosthesis usage}

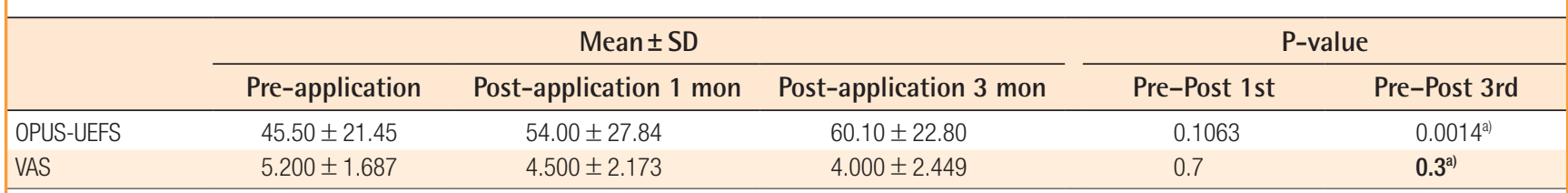

OPUS-UEFS, Orthotics Prosthetics User Survey-Upper Extremity Functional Status; VAS, visual analog scale of pain; Pre-Post 1st, between pre-application status and 1 month post-application; Pre-Post 3rd, between pre-application and 3 months post-application.

a) Statistically significant, $P<0.05$.

We sincerely apologize for the inconvenience that this may have caused. 